\title{
Does planning a different trajectory influence the choice of grasping points?
}

\author{
Dimitris Voudouris • Eli Brenner • \\ Willemijn D. Schot $\cdot$ Jeroen B. J. Smeets
}

Received: 27 November 2009/ Accepted: 23 July 2010/Published online: 7 September 2010

(c) The Author(s) 2010. This article is published with open access at Springerlink.com

\begin{abstract}
We examined whether the movement path is considered when selecting the positions at which the digits will contact the object's surface (grasping points). Subjects grasped objects of different heights but with the same radius at various locations on a table. At some locations, one digit crossed to the side of the object opposite of where it started. In doing so, it moved over a short object whereas it curved around a tall object. This resulted in very different paths for different objects. Importantly, the selection of grasping points was unaffected. That subjects do not appear to consider the path when selecting grasping points suggests that the grasping points are selected before planning the movements towards those points.
\end{abstract}

Keywords Hand paths · Grasping points ·

Grip orientation · Prehension · Moving direction

\section{Introduction}

During our daily activities, we often grasp objects with a precision grip in order to manipulate them. Some objects can only be grasped in a specific way, but most can be grasped with many combinations of grasping points. The selected grasping points depend on several properties of the target object (for an overview see Smeets and Brenner 1999) such as its location (Paulignan et al. 1997; Schot et al. 2010), orientation (Jeannerod 1981), shape (Cuijpers et al. 2004), size (Hesse and Franz 2009), centre of mass

D. Voudouris $(\bowtie) \cdot$ E. Brenner · W. D. Schot · J. B. J. Smeets Research Institute MOVE, Faculty of Human Movement Sciences, VU University, Van der Boechorststraat 9, 1081 BT Amsterdam, The Netherlands

e-mail: d.voudouris@fbw.vu.nl
(Kleinholdermann et al. 2007; Lukos et al. 2007) and the material of its surface (Fikes et al. 1994). The selected grasping points may also depend on what one intends to do with the object (for instance, a mug might be grasped differently when one wants to drink from it than when one just wants to move it). The choice of grasping points is presumably the result of somehow considering all the degrees of freedom in the joints and, probably among others, all the above-mentioned factors. The selection is presumably a compromise between postural comfort (Cuijpers et al. 2004), energetic factors (Soechting et al. 1995; Vaughan et al. 1998) and sensitivity to errors (Smeets and Brenner 1999).

An additional constraint when selecting the grasping points is that the hand should not collide with the object that is to be grasped on the way to them (Elsinger and Rosenbaum 2003; Rosenbaum et al. 2001). Therefore, the path towards the object might also be considered for the selection of the grasping points. Here, we examine whether the path is considered.

It is not evident that the path is considered. Some models, such as the equilibrium point hypothesis, explicitly assume that the path is not planned, but emerges from a selected final posture (Feldman and Levin 2009), although the path can be influenced by specifying intermediate postures (Bizzi et al. 1984). The choice of postures may optimize certain aspects of the movement (Cruse et al. 1990), but the path is not planned directly.

Other models explicitly optimize measures throughout the movement, for which one must consider the whole trajectory in advance. In that case, it may be advantageous to select different grasping points if approaching the object along a different path (Bennis and Roby-Brami 2002; Roby-Brami et al. 2000). Various postures and their success (in particular situations) could be stored, so that 
suitable movements can be recalled when a similar situation arises again (Cohen and Rosenbaum 2004; Rosenbaum et al. 2001; Vaughan et al. 2001). In that case, whether different grasping points are selected may depend on whether the situation is considered to be different. There could also be a tendency to choose grasping points that allow one to move the digits along the shortest path towards the object in order to reduce the energetic cost (Soechting et al. 1995), which directly predicts that the selected grasping points will vary with the path taken towards the object. The variety of views on motor control outlined above shows that it is not at all clear how the path and grasping points are related.

Considering the lack of experimental studies about the influence of the path on the selection of the grasping points, independent of the starting point, we here examined whether the selected grasping points are affected by the hand's path towards them. In the first experiment, a tall cylinder and a sphere were grasped at various positions on a table. The cylinder and the sphere had the same radius and both offered a wide variety of potential grasping points. We expected the cylinder not only to be grasped higher because it was taller (Lommertzen et al. 2009; Lederman and Wing 2003), but also to be grasped more carefully because it was more likely to be knocked over due to the higher location of its centre of mass. We therefore expected it to be grasped more slowly using a larger maximal grip aperture (Smeets and Brenner 1999). The main difference that we expected, however, was that subjects would move a digit over the sphere to reach a position at the other side (relative to the starting position), while they would move the digit around the tall cylinder under similar conditions (Fig. 1). They could also choose more accessible grasping positions: they could even select positions that make it unnecessary for either digit to cross to the opposite side of the object.
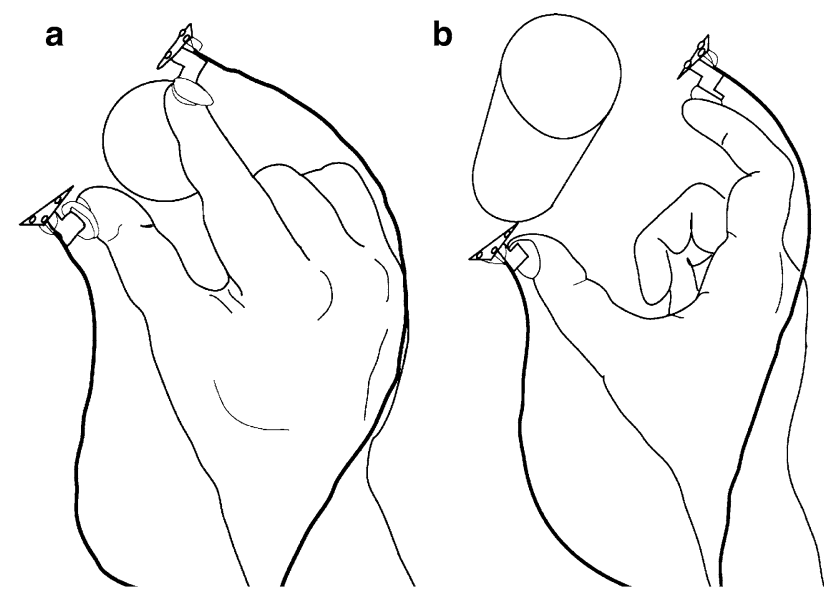

Fig. 1 Different hand paths for objects placed at the same location. Subjects move over the sphere (a), whereas they curve around the cylinder (b)
In the second experiment, we studied the influence of object height in more detail. Four cylinders of different heights were placed at two different positions on a table. Positions were chosen for which we anticipated that the digits would curve around tall cylinders and move over short ones. As object height increased, we expected the grasping points to be higher, the maximal grip aperture to be larger, and the movement time to be longer.

The main question, though, was whether the different paths that the hand takes towards objects of different heights influence the grasping points (in the horizontal plane) on these objects.

\section{Experiment 1}

Methods

\section{Subjects and apparatus}

Ten subjects, six women and four men, aged from 22 to 52 years, voluntarily participated in this study. All subjects were right-handed by self-report. Two of them were authors. The others were unaware of the purpose of the study. All participants had normal or corrected to normal vision. The experiment was part of a programme that has been approved by the local ethics committee.

Movements of the index finger and thumb were measured at a sampling rate of $250 \mathrm{~Hz}$ (resolution $0.1 \mathrm{~mm}$ ) with an Optotrak 3020 infrared tracking system with two cameras. A small rigid body with three infrared markers (see Fig. 1) was fixed to the nail of the index finger and another one was fixed to the nail of the thumb of the subject's right hand using an elastic gum.

The two objects were a black cylinder, made of polyoxymethylene $(14 \mathrm{~cm}$ height, $4.5 \mathrm{~cm}$ diameter, $323 \mathrm{~g}$ mass, with a protrusion on its bottom), and a colourful opaque glass sphere $(4.5 \mathrm{~cm}$ diameter, $123 \mathrm{~g}$ mass). A wooden board $(60 \times 52 \mathrm{~cm})$ was placed on a heightadjustable table, onto which one of the objects was placed at one of nine locations on each trial. The locations formed a $3 \times 3$ grid with $10 \mathrm{~cm}$ spacing. The locations were marked by small indentations so that the objects could easily be placed at the correct positions, and the sphere would not roll away. Two starting positions were also marked: one centred near the subject ("near") and one to the right of the grid ("side"). A schematic top view of the set-up can be seen in Fig. 2.

\section{Procedure}

Subjects stood in front of the table and its height was adjusted, if necessary, so that the task could be executed 


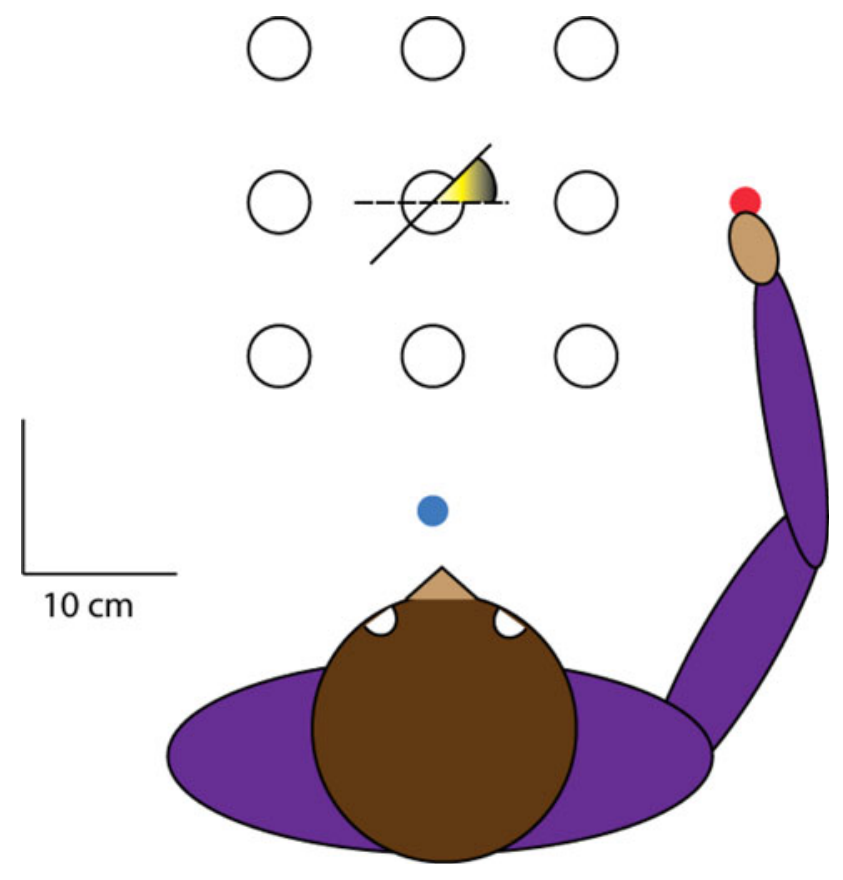

Fig. 2 Schematic top view of the set-up. The nine possible object locations are indicated by circles. The two different starting positions are shown as blue and red discs. The yellow area indicates the horizontal grip orientation

comfortably. Before each trial, subjects placed their right hand comfortably at one of the two possible starting positions, with the tips of the thumb and index finger touching each other. The experimenter indicated the starting position for each trial and placed the object at the appropriate location. He then gave a verbal signal to indicate that the subjects could start moving. Subjects were asked to grasp the object with a precision grip (holding it between index finger and thumb), to lift it and put it back at the same location, and then to move their hand back to the starting position. They could do so at their own pace. No further instructions or practice trials were given. A total of 36 conditions ( 2 starting positions $\times 2$ object types $\times$ 9 object locations) were each presented once in each of five consecutive blocks, resulting in a total of 180 trials for each subject. Within each block, the conditions were presented in a random order.

\section{Data analysis}

Prior to data collection, a calibration trial was done in which the subject held an infrared marker between thumb and index finger. This marker's position was later related to the positions of the sets of three markers on the rigid bodies attached to the fingernails. This allowed us to calculate the position in space of the part of the finger at which the handheld marker had been, from the measured positions of the markers on the rigid body. The trajectory of that part of each finger was considered to be the fingertip's trajectory towards the target object. Together, the two trajectories will be referred to as hand paths.

The speed of the hand was calculated by numerical differentiation of the average of the two digits' positions. Movement onset was defined as the first frame in which the speed of the hand was higher than $50 \mathrm{~mm} / \mathrm{s}$. The moment of the grasp was defined as the last minimum of the mean of the two digits' heights before this mean height reached its highest point (which always occurred when lifting the object). Movement time was defined as the time between movement onset and the moment of the grasp. Maximal grip aperture was defined as the maximal distance between the tips of the thumb and index finger, during the interval between movement onset and the moment of the grasp. We were mainly interested in the selected grasping points on the object, which can be expressed as the grip orientation at the moment of the grasp. Final horizontal grip orientation was defined as the orientation of the line connecting the two fingertips at the moment of the grasp, when projected onto the horizontal plane (Fig. 2). Final vertical grip orientation was defined as the angle between this horizontal projection and the line connecting the two fingertips. Horizontal grip orientation half way through the movement was calculated by determining the horizontal orientation on each trial at half the movement time.

In order to give an impression of the grasping movements, we determined average hand paths for each condition. The average positions were calculated by re-sampling the data of each digit's path to give 50 equal steps (each step corresponding to $2 \%$ of the total path length) using linear interpolation between samples. Each of the 51 coordinates was then averaged across trials.

We were interested in whether differences in hand paths would affect the choice of grasping points. We expected the largest differences between the paths towards the two objects at the near right location. In order to quantify the extent to which the hand paths were different for the two objects when they were placed at this location, we took a straight line connecting the starting and grasping points of each digit and found the maximal deviation of each digit's path from this line. We did so separately for each trial and separately for the horizontal and vertical plane. We averaged the maximal deviations across the five repetitions and compared these averages across objects and starting positions (for each digit's starting position and plane). We also determined the grip orientation half way through the movement when moving towards the near right location, averaged the values across repetitions, and compared these averages across objects and starting positions.

The main dependent variables of our study were movement time, maximal grip aperture and final grip orientation. These values were determined for each trial and 
averaged across the five repetitions for each subject and condition. The standard deviation within the five repetitions was also determined and averaged across conditions and subjects to give a measure of the variability between movements. Effects on the average dependent variables were evaluated using 9 (object locations) $\times 2$ (object types) $\times 2$ (starting positions) repeated measures analyses of variance.

Besides these analyses, we also conducted 2 (object types) $\times 2$ (starting positions) repeated measures analyses of variance for the grip orientation halfway through the movement and for the maximal vertical and horizontal deviations from the straight line connecting the starting with the grasping point of each digit. For these analyses, we only considered the trials when the object was placed at the near right location. For the deviation from the straight line, separate tests were done for the index finger and thumb.

All significant effects $(P<0.05)$ are mentioned in the results section. All significant interactions for the main dependent variables are illustrated with figures. Since we expected gradual systematic changes across object locations, we considered a graphical representation to be more informative than comparing effects at individual pairs of locations.

\section{Results}

Figure 3 shows an overview of the average grasping trajectories. The cylinder is grasped much higher than the sphere (Fig. 3b). For most object locations, the horizontal components of the digits' paths to the spheres (continuous lines in Fig. 3a) are similar to those to the cylinders (dashed lines). However, for the objects at the near right location (as seen from above) they are not. When grasping the sphere at this location from the "near" starting position, the index finger moves over the object to reach the other side. When grasping the sphere at this location starting from the "side", the thumb moves over the object (as illustrated in Fig. 1). When grasping the cylinder at this location from the "near" starting position, the index finger moves around the object to reach the other side. When grasping the cylinder at this location, starting from the "side", the thumb moves around the object. Thus, the paths are much more curved in the horizontal plane when grasping the cylinder (Fig. 4). A similar but weaker tendency can be seen at other locations. At the location at which we expected the largest effect (the near right location), the difference between the maximal horizontal deviations for the two objects is indeed significant $\left(F_{(1,9)}=35.16, \quad P<0.001, \quad\right.$ for the index finger; $F_{(1,9)}=28.49, P<0.001$, for the thumb). There was no significant difference between the maximal vertical deviations.
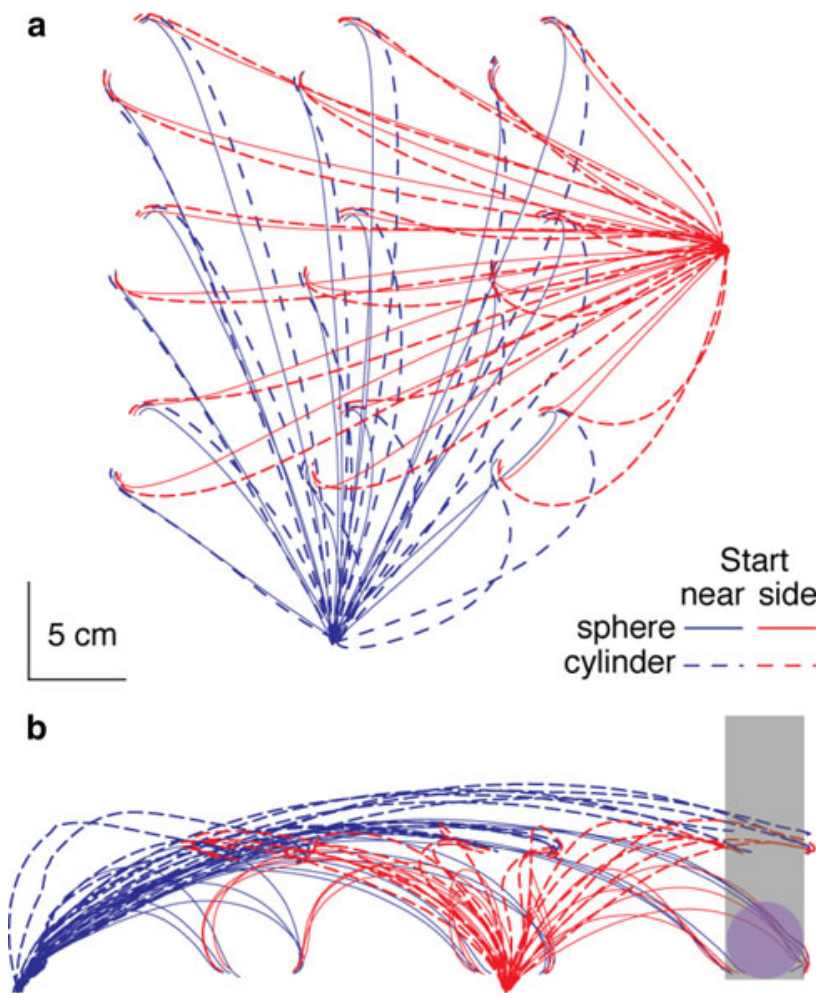

Fig. 3 Average hand trajectories in experiment 1 (mean of 5 repetitions by each of the 10 participants for each of the 36 conditions) as seen from above (a) and from the side (b)
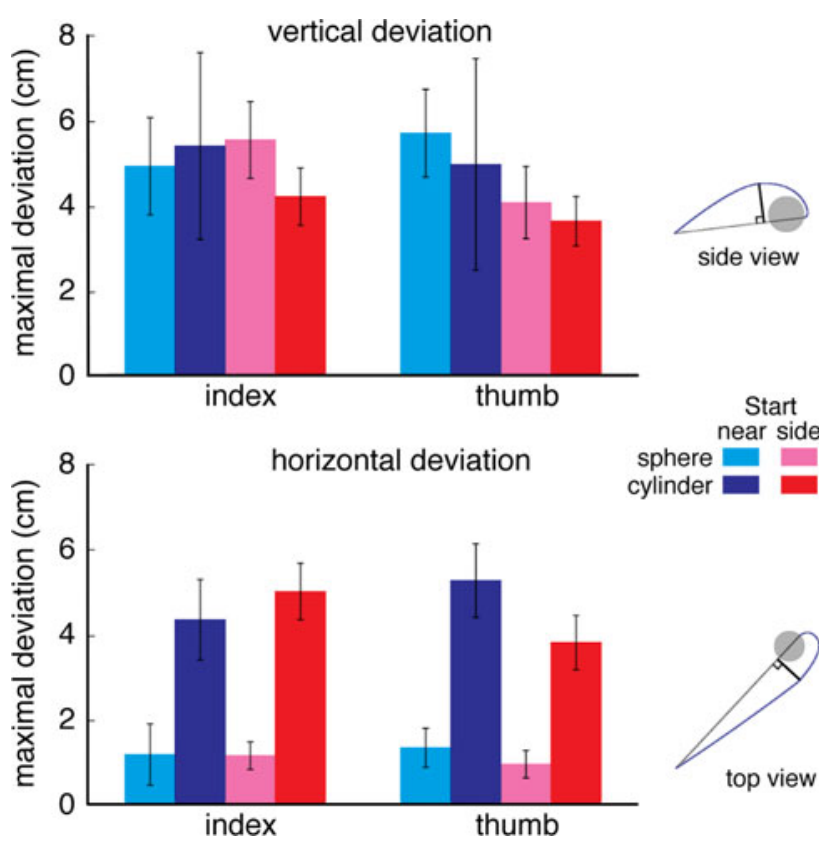

Fig. 4 Average maximal deviations of the digits from a straight line (mean and standard deviations across subjects) in experiment 1. Data for objects placed at the near right location. Values for each digit when reaching towards the two different objects from the two starting points 
Grip orientation half way through the movement to objects at the near right location also depends on the object type and starting position. There is a significant effect of starting position $\left(F_{(1,9)}=43.17, P<0.001\right.$; on average 11.9 degrees further clockwise when starting from the "near" position) as well as a significant interaction between starting position and object type $\left(F_{(1,9)}=51.17, P<0.001\right.$; the hand was oriented 12 degrees further clockwise when reaching for the cylinder when starting from the "near" position but 5 degrees further clockwise when reaching for the sphere when starting from the "side").

Despite the differences in hand paths towards the objects, the final grip orientation is very similar for both of them (no main effect of object type; Fig. 3a). Final grip orientation depends on the starting position $\left(F_{(1,9)}=28.21, P<0.001\right.$; on average it was 7.6 degrees further counter-clockwise when starting from the side). There is also a significant effect of object location $\left(F_{(8,72)}=203.18, P<0.001\right.$; see Fig. 5a). There is a significant interaction between starting position and object location $\left(F_{(8,72)}=17.13, P<0.001\right.$; Fig. 5a), and between starting position and object type $\left(F_{(1,9)}=6.23, P=0.034\right.$; inset in Fig. 5a).

To summarize, final grip orientation appears to depend on more factors than only object location. The influence of object type is negligible: subjects grasp the sphere 0.9 degrees more counter-clockwise than they do the cylinder. There is no significant interaction between starting position, object location and object type, indicating that there is no difference between object locations with regard to the critical interaction between starting position and object type. The average standard deviation within replications for the final horizontal grip orientation is 4.6 degrees.

Movement time depends on the location of the target $\left(F_{(8,72)}=28.76, P<0.001\right)$; it increases as a function of the distance from the starting position. A significant starting position by object location interaction $\left(F_{(8,72)}=12.34\right.$, $P=0.001$ ) is consistent with this (Fig. 5b). There is no main effect of object type or starting position. However, there is a significant object type by object location interaction $\left(F_{(8,72)}=3.61, P=0.001\right)$. This is mainly due to the fact that for objects at the near right location, movement times towards the cylinder were larger than the ones towards the sphere (Fig. 5c). The average standard deviation of the movement time across replications is $80 \mathrm{~ms}$.

Maximal grip aperture depends on object type $\left(F_{(1,9)}=104.04, P<0.001\right)$. The average maximal grip aperture when reaching for a cylinder is $7.1 \mathrm{~cm}$, whereas for a sphere it is only $6.2 \mathrm{~cm}$. This difference is not caused
Fig. 5 The significant interactions of experiment 1. a Starting position by object location interaction for grip orientation (the inset shows the extremely small starting position by object type interaction). b Starting position by object location interaction for movement time. c Object type by object location interaction for movement time. d Starting position by object location interaction for maximal grip aperture
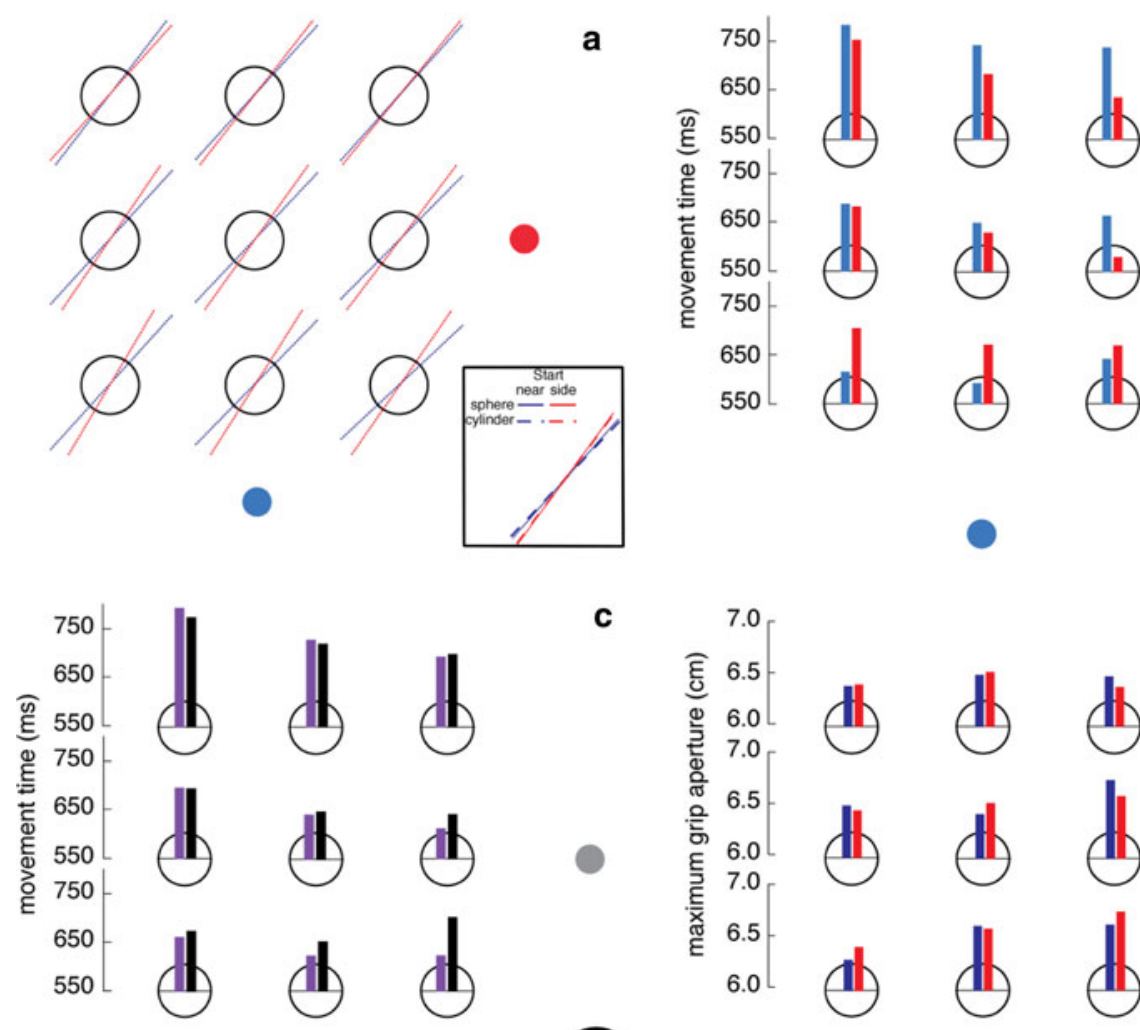

C

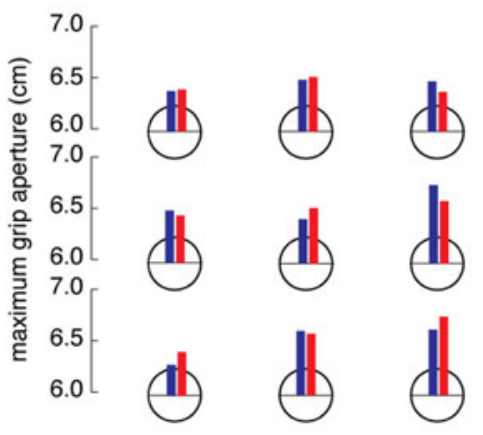

b

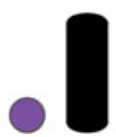


by the vertical grip orientation as the average angle that the grasping axis makes with the horizontal plane is close to zero $(3 \pm 6$ degrees and $2 \pm 7$ degrees for the cylinder and the sphere, respectively, which cannot account for more than $1 \mathrm{~mm}$ of the larger grip when grasping the cylinder than when grasping the sphere). Object location also affects maximal grip aperture $\left(F_{(8,72)}=7.66, P<0.001\right)$ : the aperture is largest for object locations near the hand. There is a significant starting position by object location interaction $\left(F_{(8,72)}=3.66, P=0.001\right.$; Fig. $\left.4 d\right)$. The average standard deviation of the maximal grip aperture across replications is $0.49 \mathrm{~cm}$.

\section{Discussion}

When the objects were placed at the locations near the hand, subjects moved along different hand paths towards the two objects. The hand curved around the cylinder and moved over the sphere to grasp it. Movement time and maximum grip aperture were also affected. Nevertheless, subjects did not choose different grasping points. Despite the different hand paths, the final grip orientation was the same for the two objects.

It is likely that the difference in object height is responsible for the different hand paths. However, the objects also differed in shape and material. It is known that friction (Burstedt et al. 1999) and fragility (Gorniak et al. 2010; Savelsbergh et al. 1996), which are related to the material of which the object is made, can affect prehension, as can the shape of the object (Cuijpers et al. 2004). In order to test whether object height was really responsible for the differences in hand paths in experiment 1 , a second experiment was carried out using cylindrical objects of different heights but of the same material.

\section{Experiment 2}

Based on the findings in experiment 1, four cylinder heights and two object locations were chosen for experiment 2; the near right location at which the difference in hand paths was the largest and the central location as a control.

\section{Methods}

Nine right-handed naïve subjects, five women and four men, aged from 24 to 41 years, volunteered to participate in this study. Except for the details mentioned below, the apparatus, procedure and data analysis were the same as in experiment 1 . Four black 4.5 -cm-diameter cylinders of different heights and mass $(4.5,7.7,10.8$ and $14.0 \mathrm{~cm}$; $105,177,249$ and $323 \mathrm{~g}$, respectively) were used. All of them were made of polyoxymethylene. On each trial, the experimenter placed one of the objects at one of two possible locations on the wooden board (the central and the near right one in Fig. 2). The starting positions were the same as in experiment 1 . A total of 16 conditions (2 starting positions $\times 4$ object heights $\times 2$ object locations) were each presented in random order in five consecutive blocks, resulting in a total of 80 trials for each subject.

In addition to the analyses of experiment 1, we also split the trials into ones in which the index finger moved over and ones in which it moved around the object, and compared the selected grasping points between the two groups of trials. Since the average vertical maximal deviation from a straight line was twice as large as the average horizontal maximal deviation, we classified trials in which the ratio between the maximal vertical deviation and the maximal horizontal deviation was equal or higher than two as "move-over" trials. The rest were classified as "curvearound" trials.

\section{Results}

Figure 6 shows the hand paths towards the four different objects, as seen from above (a) and from the side (b). Hand paths end at a higher position as the height of the object increases. When the object is placed at the near right location, the pattern of the hand path's curvature is consistent with, and extends, that of experiment 1: the taller the object the more curved the hand path (Fig. 7). This pattern is not evident for the object at the other location (not shown). At the near right location, the differences in maximal horizontal deviation between the objects are significant $\left(F_{(3,24)}=9.86, P<0.001\right.$ for the index finger; $F_{(3,24)}=7.97, P<0.001$ for the thumb; Fig. 7), whereas those in the maximal vertical deviation are not.

Since the cylinder height at which subjects switched from moving over to moving around the object differed across subjects, we also averaged the data on the basis of whether the hand moved over or around the object, irrespective of the object height. Figure 8 shows that moving around rather than over the objects results in a clear difference in the direction in which the digits approach the objects but does not result in different grasping points being chosen.

Altogether the results are very similar to those of experiment 1. Hand paths are affected by object height, but the grasping points are very similar when reaching for different objects (Fig. 6a). Final grip orientation is 8.4 degrees further counter-clockwise when starting from the side $\left(F_{(1,8)}=100.03, P<0.001\right)$. There is also a significant effect of object location $\left(F_{(1,8)}=97.08\right.$, $P<0.001$; further counter-clockwise for the central 
a

$5 \mathrm{~cm}$

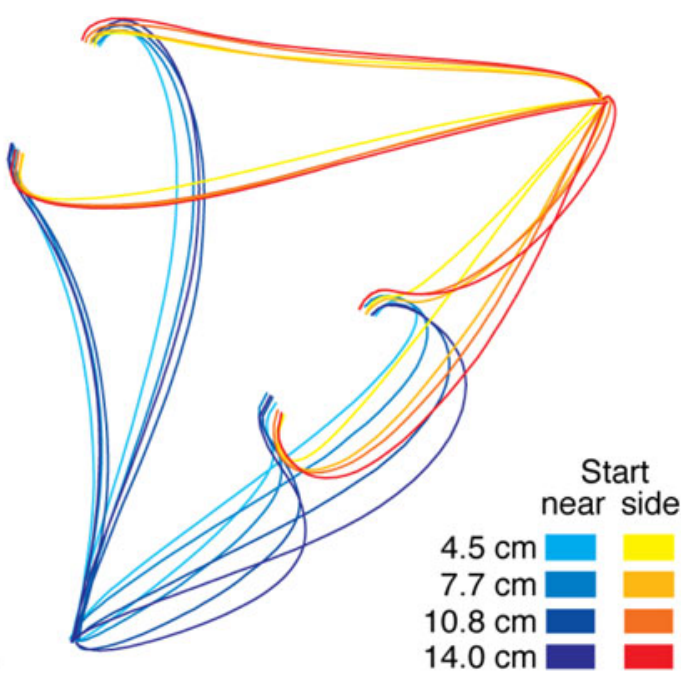

b

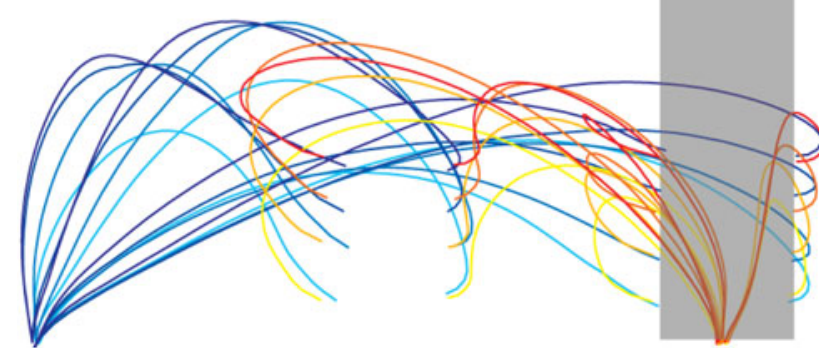

Fig. 6 Average hand trajectories in experiment 2 (mean of 5 repetitions by each of the 9 participants for each of the 16 conditions) as seen from above (a) and from the side (b) object location) and a significant interaction between starting position and object location $\left(F_{(1,8)}=26.63\right.$, $P<0.001$; Fig. 9a). Note that for the final grip orientation there is neither a significant main effect of object height nor a significant interaction involving object height. The average standard deviation within replications for the final horizontal grip orientation is 4.4 degrees.

Movement time depends on object location $\left(F_{(1,8)}=24.73, P=0.001\right)$. It is larger for objects placed at the near right location than for those at the central location. Moreover, there is a significant object type by object location interaction $\left(F_{(3,24)}=4.68, \quad P<0.05\right.$; Fig. 9b): At the near right locations movement times increase with object height, whereas at the central location they decrease slightly. The standard deviation of the movement time within replications is $100 \mathrm{~ms}$.

Maximal grip aperture depends on the object type, with larger apertures when reaching for taller objects $\left(F_{(3,24)}=27.35, \quad P<0.001 ; 7.24 \mathrm{~cm}( \pm 0.83), 7.44 \mathrm{~cm}\right.$ $( \pm 0.80), 7.70 \mathrm{~cm}( \pm 0.84)$ and $7.83 \mathrm{~cm}( \pm 0.85)$, for the $4.5,7.7,10.8$ and $14.0 \mathrm{~cm}$ high cylinders, respectively). Object location also affects maximal grip aperture, with larger grips for objects at the near right location $\left(F_{(1,8)}=12.78, \quad P<0.05\right)$. There is also a significant starting position by object location interaction $\left(F_{(1,8)}=10.12, P<0.05\right.$; Fig. 9c). The average standard deviation within replications for the maximal grip aperture is $0.6 \mathrm{~cm}$.
Fig. 7 Average maximal deviations of the digits from a straight line (mean and standard deviations across subjects) in experiment 2. Data for objects placed at the near right location. Values for each digit when reaching towards the four different objects from the two starting points
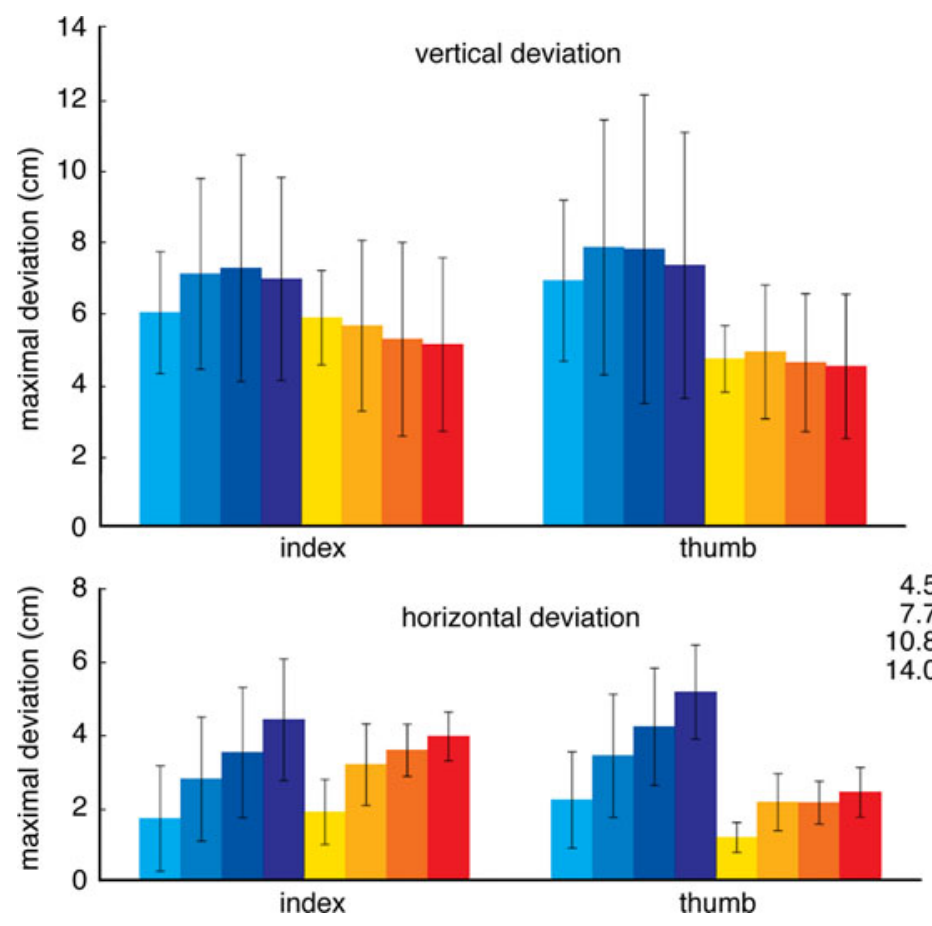

side view

Start near side $4.5 \mathrm{~cm}$ $7.7 \mathrm{~cm}$ $0.8 \mathrm{~cm}$ $4.0 \mathrm{~cm}$

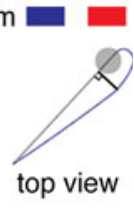




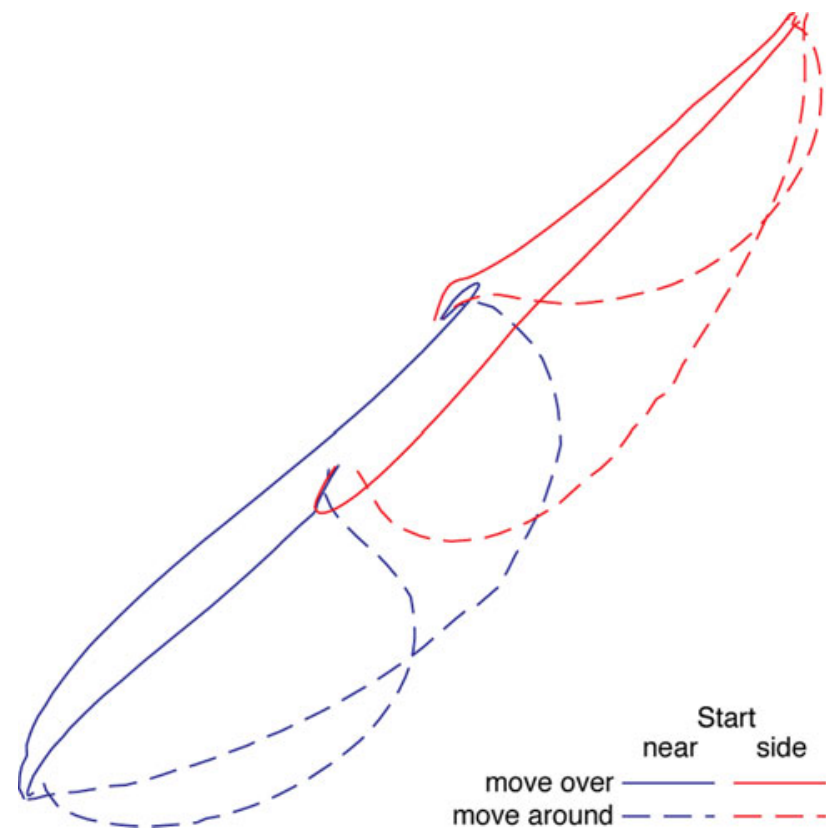

Fig. 8 Top view of average hand trajectories in experiment 2. Movements were selected on the basis of whether the hand moved over or around the object. Despite the large differences in hand trajectories, the grasping points are very similar

\section{Discussion}

As in experiment 1, subjects selected different hand paths towards the different objects. The curvature of the digits around the objects clearly increased as a function of object height. This did not affect the grasping points. Altogether, the significant effects in experiment 2 were very similar to those of experiment 1 , suggesting that object height was responsible for the differences in performance in both experiments.

\section{General discussion}

Our main finding is that the same grasping points were selected when moving towards the object in clearly different ways. Although this appears to be consistent with equilibrium point control or some other control that selects a final posture without considering the path, this interpretation does not directly account for all our findings. The fact that the movement path does depend on object type indicates that not only the endpoints determine the movement path. When a tall object was placed near the hand, one of the digits curved around it. When a short one was placed at the same location, one of the digits moved over it, although it could have curved around it in the same way as it did for the tall object. The difference presumably arises from a tendency to move the hand along the most advantageous path. Determining this path is not trivial. Many factors have to be considered. Selecting a path may involve considering stored postures and selecting the most appropriate one for a specific situation (Rosenbaum et al. 2001). If so, having a different object height might be recognized as a different situation. Whatever the control mechanism, in this study the curvature of the path around the tall objects (when the objects were placed near the hand) is probably especially large, because subjects also had to avoid hitting the object with the rigid bodies containing the markers (see Fig. 1). Note that the markers' rigid bodies do not constrain the choice of grasping points; they only constrain the digits' paths.

Subjects grasped the tall objects higher than the short ones. This is not surprising because the centre of mass of the former is higher, and it is advantageous to grasp objects above their centre of mass (Lederman and Wing 2003). As the tall objects are more likely to be knocked over, subjects were expected to move more carefully towards them to make sure not to collide with them (Lommertzen et al. 2009; Smeets and Brenner 1999). A more careful approach was reflected in the larger grip aperture when grasping tall objects, but there was no increase in movement time, except when the object was near the hand, in which case the path towards it was more curved and thus also longer, so the increase in movement time cannot be attributed to a more careful approach.

Our question was whether the grasping points would differ if subjects took a different path to the object. Rather than asking subjects to move towards the object in different ways, which subjects may interpret as an instruction to use different grasping points, we used different objects towards which we expected subjects to move in different ways; as they did. The
Fig. 9 The significant interactions of experiment 2. a Starting position by object location interaction for grip orientation, $\mathbf{b}$ object type by object location interaction for movement time, and c starting position by object location interaction for maximal grip aperture a

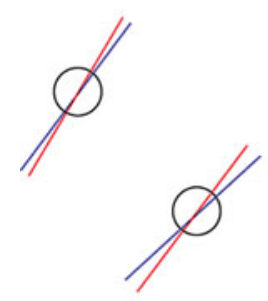

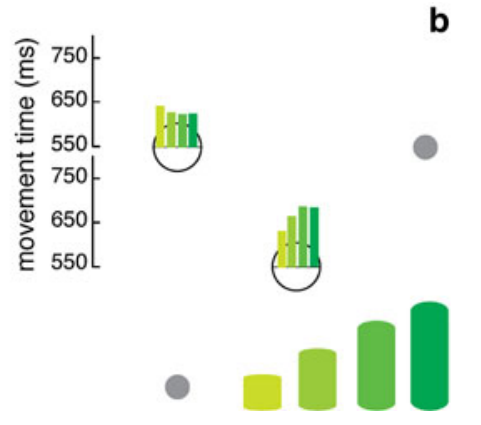

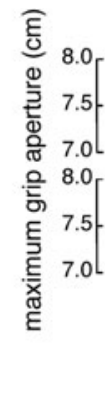

c
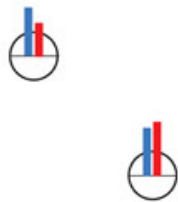
differences between the paths arose because the object itself obstructs the movement, rather than through additional objects. We considered it to be important for the hand paths towards the objects to differ due to optimization factors and not because we introduced additional constraints (such as introducing additional obstacles) that could affect the selection of the grasping points directly. Thus, we wanted to be sure that only the different hand paths could be responsible for any difference in the grasping points.

Some have previously argued that the final posture is independent of the selected hand path (Grea et al. 2000), whereas others have argued for a dependence on it (Rosenbaum et al. 2001). Our study supports the former position. However, this does not mean that subjects will never vary their grasping points without being forced to do so. If there is a risk of ending in an uncomfortable posture when manipulating the grasped object, people are willing to grasp the object with an uncomfortable posture in order to end up in a comfortable one (Rosenbaum et al. 1992). These findings illustrate that many factors are considered when determining the grasping points. We show that the path towards the object is not one of these factors.

On the basis of earlier studies (Elsinger and Rosenbaum 2003; Roby-Brami et al. 2000), one could have expected the grasping points to depend on the optimization of the whole path rather than only on the final posture, and therefore for the grasping points to depend on the hand's path. In our study, the grasping points were influenced by the different starting positions, as in the study of Roby-Brami et al. (2000), although the effect was very small (see also Schot et al. 2010). This seems to suggest that the direction in which the hand approaches the object matters, but the very different hand paths towards the objects when placed near the hand did not affect the grasping points at all, so presumably the influence of the starting position on the selection of grasping points is primarily determined by the posture before the movement rather than by the movement itself.

As the different movement trajectories did not affect the grasping points, we could assume that subjects selected the grasping positions on the object before deciding how to move towards them. Subjects seem to choose where to place their digits on the basis of the final posture, independently of selecting the best path towards the grasping points. The selection of the grasping points is presumably made before starting to move (Hesse et al. 2008; Grea et al. 2000), although the choice can change during the movement if the object is perturbed (Desmurget and Prablanc 1997; Desmurget et al. 1995; Grea et al. 2000).

Acknowledgments The research leading to these results has received funding from the European Community's Seventh Framework Programme FP7/2007-2013 under grant agreement number 214728-2.
Open Access This article is distributed under the terms of the Creative Commons Attribution Noncommercial License which permits any noncommercial use, distribution, and reproduction in any medium, provided the original author(s) and source are credited.

\section{References}

Bennis N, Roby-Brami A (2002) Coupling between reaching movement direction and hand orientation for grasping. Brain Res 952:257-267

Bizzi E, Accornero N, Chapple W, Hogan N (1984) Posture control and trajectory formation during arm movement. J Neurosci 4:2738-2744

Burstedt MKO, Flanagan JL, Johansson RS (1999) Control of grasp stability in humans under different frictional conditions during multidigit manipulation. J Neurophysiol 82:2392-2405

Cohen RG, Rosenbaum DA (2004) Where grasps are made reveals how grasps are planned: generation and recall of motor plans. Exp Brain Res 157:486-495

Cruse H, Wischmeyer E, Brüwer M, Brockfeld P, Dress A (1990) On the cost functions for the control of the human arm movement. Biol Cybern 62:519-528

Cuijpers RH, Smeets JBJ, Brenner E (2004) On the relation between object shape and grasping kinematics. J Neurophysiol 91:2598-2606

Desmurget M, Prablanc C (1997) Postural control of three-dimensional prehension movements. J Neurophysiol 77:452-464

Desmurget M, Prablanc C, Rossetti Y, Arzi M, Paulignan Y, Urquizar C, Mignot JC (1995) Postural and synergic control for threedimensional movements of reaching and grasping. J Neurophysiol 74:905-910

Elsinger CL, Rosenbaum DA (2003) End posture selection in manual positioning: evidence for feedforward modeling based on a movement choice method. Exp Brain Res 152:499-509

Feldman AG, Levin MF (2009) The equilibrium-point hypothesisPast, present and future. In: Sternad D (ed) Progress in motor control. Springer, US, pp 699-726

Fikes TG, Klatzky RL, Lederman SJ (1994) Effects of object texture on precontact movement time in human prehension. J Mot Behav 26:325-332

Gorniak SL, Zatsiorsky VM, Latash ML (2010) Manipulation of a fragile object. Exp Brain Res 202:413-430

Grea H, Desmurget M, Prablanc C (2000) Postural invariance in three-dimensional reaching and grasping movements. Exp Brain Res 134:155-162

Hesse C, Franz VH (2009) Corrective processes in grasping after perturbations of object size. J Mot Behav 41:253-273

Hesse C, De Grave DDJ, Franz VH, Brenner E, Smeets JBJ (2008) Planning movements well in advance. Cogn Neuropsychol 25:985-995

Jeannerod M (1981) Intersegmental coordination during reaching at natural vision objects. In: Long J, Baddeley A (eds) Atten and perform IX. Erlbaum, Hillsdale, pp 153-169

Kleinholdermann U, Brenner E, Franz VH, Smeets JBJ (2007) Grasping trapezoidal objects. Exp Brain Res 180:415-420

Lederman SJ, Wing AM (2003) Perceptual judgement, grasp point selection and object symmetry. Exp Brain Res 152:156-165

Lommertzen J, Costa e Silva E, Cuijpers RH, Meulenbroek RGJ (2009) Collision-avoidance characteristics of grasping. Early sign in hand and arm kinematics. Anticipatory behaviour in adaptive learning Systems. Lect Notes Comput Sci 5499(2009):188-208 
Lukos J, Ansuini C, Santello M (2007) Choice of contact points during multidigit grasping: effect of predictability of object centre of mass location. J Neurosci 27:3894-3903

Paulignan Y, Frak VG, Toni I, Jeannerod M (1997) Influence of object position and size on human prehension movements. Exp Brain Res 114:226-234

Roby-Brami A, Bennis N, Mokhtari M, Baraduc P (2000) Hand orientation for grasping depends on the direction of the reaching movement. Brain Res 869:121-129

Rosenbaum DA, Vaughan J, Barnes HJ, Jorgensen MJ (1992) Time course of movement planning: selection of handgrips for object manipulation. J Exp Psychol Learn Mem Cogn 18:1058-1073

Rosenbaum DA, Vaughan HJ, Meulenbroek RJ, Jansen C (2001) Posture-based motion planning: applications to grasping. Psychol Rev 108:709-734

Savelsbergh GJP, Steenbergen B, van der Kamp J (1996) The role of fragility information in the guidance of the precision grip. Hum Mov Scie 15:115-127
Schot WD, Brenner E, Smeets JBJ (2010) Posture of the arm when grasping spheres to place them elsewhere. Exp Brain Res 204:163-171

Smeets JBJ, Brenner E (1999) A new view on grasping. Mot Control 3:237-271

Soechting JF, Buneo CA, Herrmann U, Flanders M (1995) Moving effortlessly in three dimensions: does Donders' law apply to arm movement? J Neurosci 15:6271-6280

Vaughan J, Rosenbaum DA, Harp CJ, Loukopoulos LD, Engelbrecht S (1998) Finding final postures. J Mot Behav 30:273-284

Vaughan J, Rosenbaum DA, Meulenbroek RGJ (2001) Planning reaching and grasping movements: the problem of obstacle avoidance. Mot Control 5:116-135 